III.

\title{
A Case of Abscess of the Ovary following an Attack of Puerperal Septicæmia.
}

\author{
By A. Lovisw McTlroy, M.D., \\ Gynacologist to the Victoria Infrmary and District Physician to \\ the Maternity Hospital, Glasgow.
}

THE patient, Mrs. C, unipara, aged 25, was sent into the Victoria Infirmary from the Lanark Fever Mospital, and I am indebted to Dr. Watt of that institution for the notes of the case. In February of 1906, she was delivered of a child without instrumental aid, but the third stage of labour was prolonged, the placenta having to be removed in picces, some post partum hæmorrhage taking place. Sepsis having taken place, the patient was transferred to the fever hospital for further treatment. On her admission the uterus was curetted and found to contain some placental débris, and on examination of the pelvis a fulness was felt in the right vaginal fornix. $\Lambda$ few days later this swelling became more marked and an incision was made in the vagina and some pus had evacuated. The patient showed marked improvement for several weeks, but the temperature became intermittent in character so she was sent to the Victoria Infirmary for further operative measures.

The patient was admitted to the ward on the 27th April and the condition was as follows:-

The patient is pale and somewhat emaciated, and looks as if she were suffering pain. The temperature is $101^{\circ} \mathrm{F}$., and the pulse quick. On examination of the abdomen a rounded swelling is seen occupying the hypogastric region, extending to within $2 \frac{1}{2}$ in. of the umbilicus and measuring $5 \mathrm{in}$. in its transverse diameter. On palpation there is some tenderness over the tumour, more marked in the left iliac region; there is slight movement from side to side but no fluctuation can be made out. There does not appear to be any infiltration of the surrounding cellular tissues, the tumour having the appearance of that of a pregnant uterus and giving a dull note on percussion over its whole area. Owing to the tenderness complained of by the patient, I made the vaginal examination under an ansthetic and found the tumour mass to be continuous with the cervix; the appendages could not be made out separately from the swelling. In front of the tumour and just above the region of the internal os was a small nodule about the size of a hen's egg, which could not be moved separately from the larger mass but seemed to be incorporated with 
it. The sound passed $3 \frac{1}{2} \mathrm{in}$. in the normal direction. The diagnosis of a fibroid condition of the uterus was made, but owing to the temperature still remaining high I decided to postpone operating until the patient was in a more favourable condition. She was kept in the ward for three weeks and was treated with hot douches, etc., with $3 i$ doses of Easton's syrup before food and gr. iii doses of reduced iron after. The temperature became normal and she was discharged and told to return to the hospital for further cxamination. I saw her several times subsequently, and at first she appeared to be much better, but the pain becoming very severe in the left side, I took her in to the ward again, with the object of removing the uterus.

On her second admission, September 25th, the temperature and pulse were normal, and the patient appeared to be much better than at her former visit. The swelling in the abdomen was, however, larger in size, cxteuding up to the nmbilicus and lying somewhat over to the left side, it was rounded in outline and of firm consistence, no fluctuation being made out. There was considerable tenderness in the suprapubic and left iliac regions. The whole mass seemed fixed in the abdominal cavity and could not be moved separately from the cervix.

On the 2nd October I opened the abdomen with Dr. MeLellan assisting. On incision a large tumour, having the appearance of an ovarian cyst, was found filling the whole abdomen and about the size of a football. In front of the cyst and closely adherent to its wall was the uterus, slightly enlarged and thickened. The upper border and whole lower surface of the tumour was closely adherent to the omentum and bowel. Removal as a whole was found to be impossible, owing to the dense peritonitic adhesions, and after careful covering of the edges of the wound and surrounding structures with gauze, the cyst was aspirated with a small trocar and cannula, and the contents, when evacuated, were found to consist of pus.

The margins of the puncture were brought together with forceps and the opening closed. The adhesions were separated with great difficulty in the region of the rectum, being so dense that the cyst wall was torn and some of the contents escaped. The pedicle was dealt with in the usual manner and the cyst removed. 'The tumour was found to have its origin in the left ovary; the right ovary and tube were somewhat congested but otherwise normal and were not removed. The abdomen was closed in two layers, through and through sutures of salmon gut, and cat-gut sutures through the sheath of the recti muscles.

The pus was examined for micro-organisms, and after some hours cultures of streptococei were obtained.

The patient did well for twenty-four hours, but signs of peritonitis appeared, and she died thirty-six hours after operation, the temperature rising to $104^{\circ} \mathrm{F}$. 
I am much indebted to Dr. Eadie, house-surgeon, for his careful notes of the case, and for the preparation of the slides and culture tubes.

\section{Remarks.}

The specimen, on examination after removal, was found on its external aspect to have the appearance of an ovarian cyst, being smooth and rounded in outline. The walls of the cyst, on section, measured $\frac{1}{4}$ in. in thickness, and appeared fibrous in character. The lining membrane had the corrugated appearance eharacteristic of pus cavities. The Fallopian tube was thickened but did not contain pus, and on microscopical examination showed only slight erosion of its epithelial surface. 'Taking into consideration the history of the case, the absence of pus from both tubes, the infection which occurred in the uterus at the time of removal of the placenta must have extended by means of the blood stream, or by the lymphatics in the broad ligament, and not by means of the Fallopian tubes.

At the first operation for the evacuation of the pus in the right vaginal fornix, the cellular tissue was found to be involved on the right side alone, and it is hardly possible that the right tube and ovary, if they were the seat of infection at that time, could recover so rapidly as to show almost normal appearances at the time of opening the abdomen. Most probably the infection travelled by means of the cellnlar tissue on the right side, and the pus being set free by incision the discharge soon ceased. On the left side the streptococci must have invaded the ovary by means of the blood stream or by the lymphatics of the broad Jigament, the pus contents being shut off from the surrounding tissues by means of the thickened tunica albuginea. Although no history could be obtained of any swelling in the abdomen previous to the onset of pregnancy, it is possible that a pre-existing ovarian cyst became infected at the time of labour, just as we have records of infection of ovarian tumours occurring during an attack of typhoid fever. 